\title{
Functional Dissociation within the Entorhinal Cortex for Memory Retrieval of an Association between Temporally Discontiguous Stimuli
}

\author{
Mark D. Morrissey, ${ }^{1,3}$ Geith Maal-Bared, ${ }^{1}$ Sinead Brady, ${ }^{1}$ and Kaori Takehara-Nishiuchi ${ }^{1,2,3}$ \\ Departments of ${ }^{1}$ Psychology and ${ }^{2}$ Cell and Systems Biology, and ${ }^{3}$ Neuroscience Program, University of Toronto, Toronto, Ontario M5S 3G3, Canada
}

\begin{abstract}
Anatomical connectivity and single neuron coding suggest a segregation of information representation within lateral (LEC) and medial (MEC) portions of the entorhinal cortex, a brain region serving as the primary input/output of the hippocampus and maintaining widespread connections to many association cortices. The present study aimed to expand this idea by examining whether these two subregions differentially contribute to memory retrieval for an association between temporally discontiguous stimuli. We found that reversible inactivation of the LEC, but not the MEC, severely impaired the retrieval of the recently and remotely acquired memory in rat trace eyeblink conditioning, in which a stimulus-free interval was interposed between the conditioned and unconditioned stimulus. Conversely, inactivation of the LEC had no effect on retrieval in delay eyeblink conditioning, where two stimuli were presented without an interval. Therefore, the LEC, but not the MEC, plays a long-lasting role in the retrieval of a memory for an association between temporally discontiguous stimuli.
\end{abstract}

\section{Introduction}

Studies in humans, nonhuman primates, and rodents highlight the importance of the hippocampus and its surrounding rhinal cortices in the formation and retrieval of episodic memory (Scoville and Milner, 1957; Squire and Zola-Morgan, 1991). Among the rhinal cortices, the entorhinal cortex serves as the primary input/output structure for the hippocampus, and can be divided into caudomedial (medial entorhinal cortex, MEC) and rostrolateral (lateral entorhinal cortex, LEC) regions. The MEC receives a greater proportion of projections from visual and visuospatial cortices, whereas the LEC receives stronger inputs from multiple association cortices arriving largely through the perirhinal cortex (Canto et al., 2008; Agster and Burwell, 2009). Consistent with this segregation in anatomical connectivity, activities of neurons in the MEC are modulated by spatial information (i.e., grid cells, Fyhn et al., 2004; Hafting et al., 2005) and are necessary for spatial memory formation and retrieval (Steffenach et al., 2005; Gaskin and White, 2010). In contrast, such spatially responsive cells are nearly absent in the LEC (Hargreaves et al., 2005).

The functional role of the LEC is less established; however, it may be involved in memory associations requiring sensory information

Received Oct. 14, 2011; revised Jan. 5, 2012; accepted Jan. 5, 2012

Author contributions: K.T.-N. designed research; M.D.M., G.M.-B., S.B., and K.T.-N. performed research; M.D.M. analyzed data; M.D.M. and K.T.-N. wrote the paper.

This work was supported by a Natural Sciences and Engineering Research Council of Canada (NSERC) Discovery grant, Canada Foundation for Innovation Leaders Opportunity Fund (K.T.-N.), NSERC graduate fellowship (M.D.M.), and NSERC undergraduate student research awards program (G.M.-B.). We thank Dr. Sara J. Shettleworth for equipment support.

Correspondence should be addressed to Kaori Takehara-Nishiuchi, Department of Psychology, University of Toronto, Sidney Smith Hall Room 4033, 100 St. George Street, Toronto, Ontario M5S 3G3, Canada. E-mail: takehara@psych.utoronto.ca.

DOI:10.1523/JNEUROSCI.5227-11.2012

Copyright $\odot 2012$ the authors $\quad 0270-6474 / 12 / 325356-06 \$ 15.00 / 0$ maintenance. The LEC receives highly processed sensory information from the perirhinal cortex (Canto et al., 2008; Agster and Burwell, 2009), and upon stimulation entorhinal neurons show persistent spiking after stimulus termination in vitro, lasting from tens of seconds to minutes in MEC neurons, and for an indefinite period of time in LEC neurons (Klink and Alonso, 1997; Tahvildari et al., 2007). Lesions and pharmacological manipulations to the entorhinal cortex, including medial and lateral portions, impair memory paradigms that require the maintenance of sensory information over a temporal delay (Otto and Eichenbaum, 1992; Ryou et al., 2001; Esclassan et al., 2009), however no study has investigated whether this function can be mediated specifically by the LEC. To address this point, the present study examined the effect of reversible inactivation of the LEC or MEC on memory expression in two classical eyeblink conditioning paradigms. We found that inactivation of the LEC, but not the MEC, impaired memory expression in the trace paradigm, where an auditory stimulus (conditioned stimulus, CS) and electric shock to the eyelid (unconditioned stimulus, US) were paired with an interval between them. In contrast, LEC inactivation did not impair memory expression in the delay paradigm, in which the CS coterminated with the US.

\section{Materials and Methods}

\section{Subjects}

Subjects were 24 male Long-Evans rats obtained from Charles River Laboratories at 70 days of age. Rats were housed individually in clear Plexiglas cages in a colony room with a $12 \mathrm{~h}$ reversed light/dark cycle (lights off at 10:00 A.M.) and given ad libitum access to food and water. All experimental procedures were in conformity with the guidelines and procedures outlined by the NIH Guide for the Care and Use of Laboratory Animals (Publication No. 85-23, revised 1985) and the Canadian Council on Animal Care, and were approved by the University of Toronto Animal Care Committee. 
A
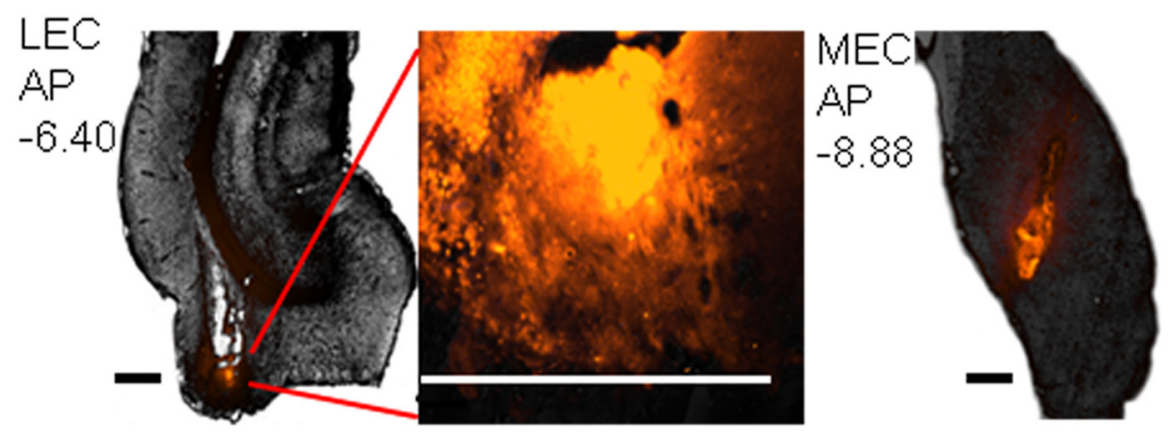

B
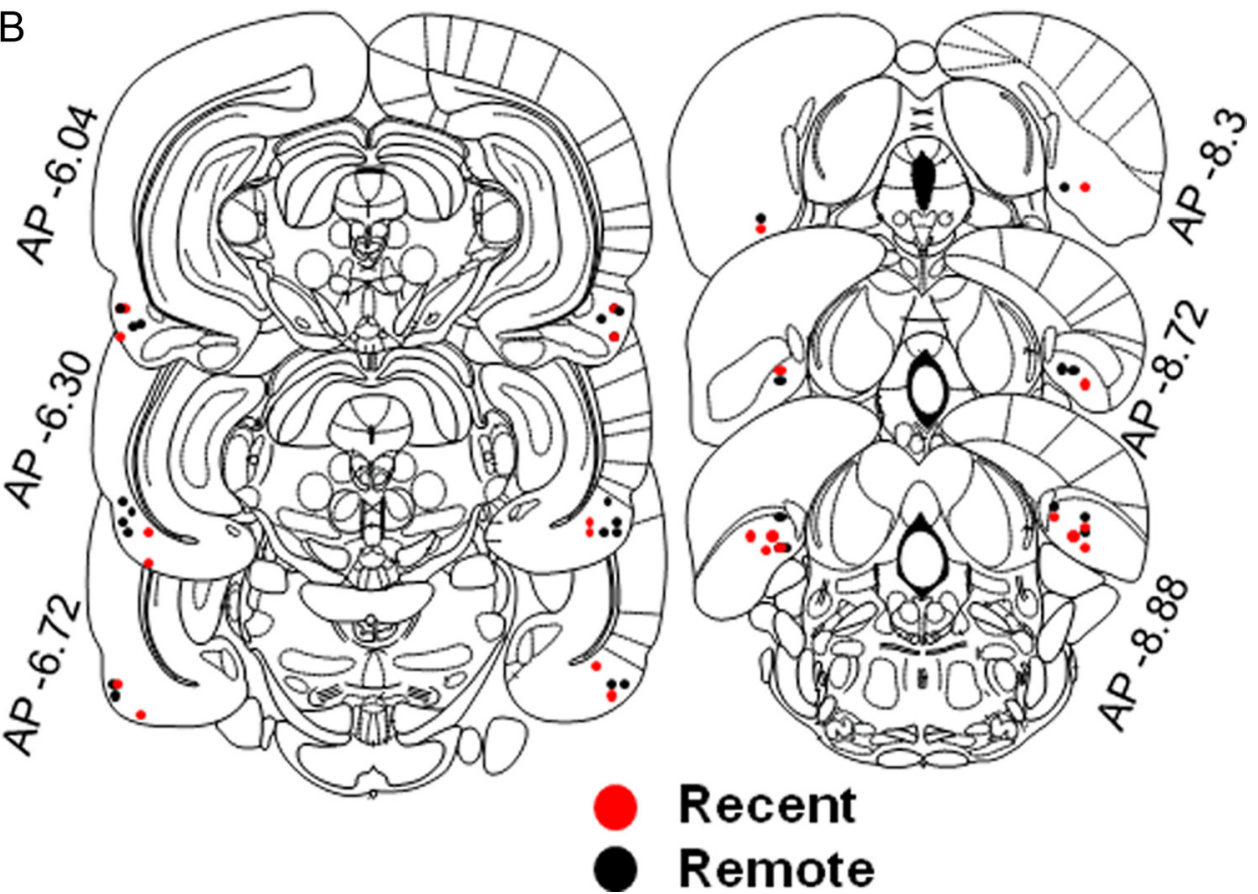

Figure 1. Cannula locations and spread of muscimol. $A$, A fluorescent tagged muscimol conjugate infused into the lateral (LEC, left) and medial (MEC, right) entorhinal cortex. Spread was confined to a maximal $1 \mathrm{~mm}$ range. A magnified view of a LEC infusion site is also provided (center). Scale bars, $600 \mu \mathrm{m}$. $\boldsymbol{B}$, Histological reconstructions of cannula locations in the LEC (left) and MEC (right) of the animals included in the analyses. In 18 animals ( $1 \mathrm{~d}$ retention, Recent, $n=8$, red circles; 1 month retention, Remote, $n=10$, black circles), the cannulae were bilaterally positioned in the LEC, and of those 18 animals, 12 also had bilateral cannulae in the MEC (Recent, $n=6$; Remote, $n=6$ ). Images were adapted from Paxinos and Watson, Copyright Elsevier (1998) with permission.

\section{Design}

All rats were trained in a trace eyeblink conditioning paradigm, followed by a $6 \mathrm{~d}$ period of retention testing. Before conditioning, rats were randomly assigned to two groups; the Recent group received retention sessions $1 \mathrm{~d}$ after conditioning, and the Remote group was tested on retention 1 month after conditioning. Following the last retention session, all rats underwent one acquisition and two retention sessions in a delay eyeblink conditioning paradigm.

\section{Surgery}

Rats received one of two surgery protocols based on group assignment: (1) rats in the Recent group were implanted with electrodes for electromyogram (EMG) and periorbital shock, and guide cannulae for drug infusion, before acquisition sessions $(n=12)$; (2) rats in the Remote group received electrode implantation only, then electrode replacement and guide cannulae implantation 1 week before retention testing. Surgeries followed similar protocols as reported previously (Takehara et al., 2003). Briefly, under anesthesia with isoflurane $(1-1.5 \%$ by volume in oxygen at a flow rate of $1.5 \mathrm{~L} / \mathrm{min}$; Holocarbon Laboratories) rats had four Teflon-coated stainless-steel wires implanted subcutaneously in the obicularis occuli muscle of the upper left eyelid for EMG recording and periorbital shock delivery, and another wire implanted into the neck for reference; all wires were attached to a small connector cap. Small burr holes were drilled bilat- erally on the skull, and guide cannulae (Plastics One) were lowered at the following coordinates: For the LEC: -6.45 anteroposterior (AP), \pm 6.65 mediolateral (ML), and $-8.5 \mathrm{~mm}$ dorsoventral (DV) to breg$\mathrm{ma}$; for the MEC: $-8.88 \mathrm{AP}, \pm 4.7 \mathrm{ML}$, and $-5.3 \mathrm{~mm}$ DV. All coordinates were adjusted from the rat atlas of Paxinos and Watson (1998) based on histology in our pilot study. The cannulae and connector cap were then secured to the skull with dental acrylic and stainless-steel screws, and cannulae were capped with dummy stylets.

\section{Intracerebral drug infusion}

Before each retention session, rats were placed in plastic restrainers (Stoelting) and received bilateral infusions in the LEC, MEC, or both, at a speed of $0.5 \mu \mathrm{l}$ per min of either muscimol [Muscimol $\mathrm{HBr}$, Sigma, $0.5 \mu \mathrm{g}$ in $0.5 \mu \mathrm{l}$ of artificial CSF (aCSF)] or $0.5 \mu \mathrm{l}$ of aCSF using a microinfusion pump (Harvard Apparatus). On each of six retention days, individual rats received one of six possible infusions of either muscimol (Mus) or aCSF in the MEC, LEC, or both: [(1) MusLEC; (2) Mus-MEC; (3) Mus-LEC and MEC; (4) aCSF-LEC; (5) aCSF-MEC; (6) aCSF-LEC and MEC] at least 10 min before the start of the session. Infusion schedules were generated randomly, with all rats receiving each infusion only once. The experimenter was blind to the infusion schedules. 


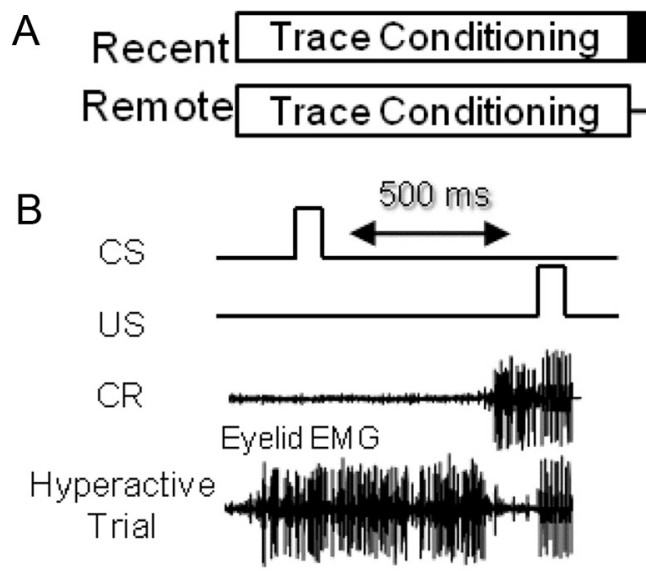

C

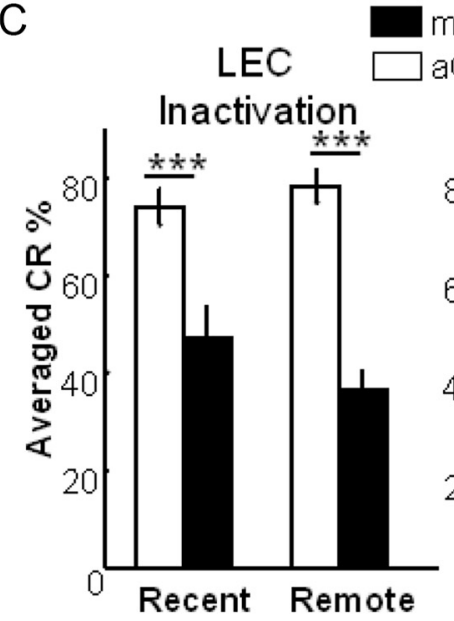

muscimol

Retention

\section{One month}

Retention

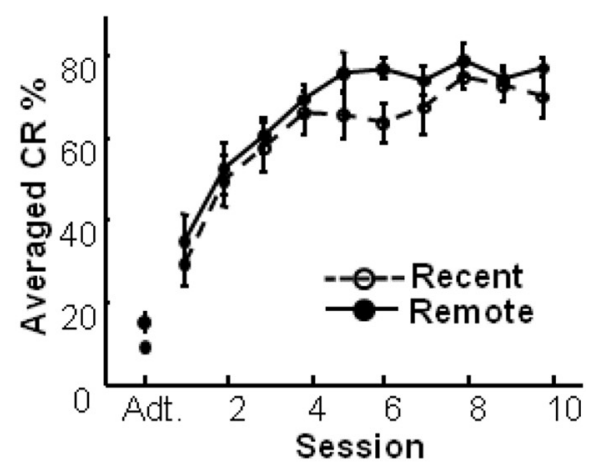

MEC Inactivation

LEC+MEC Inactivation
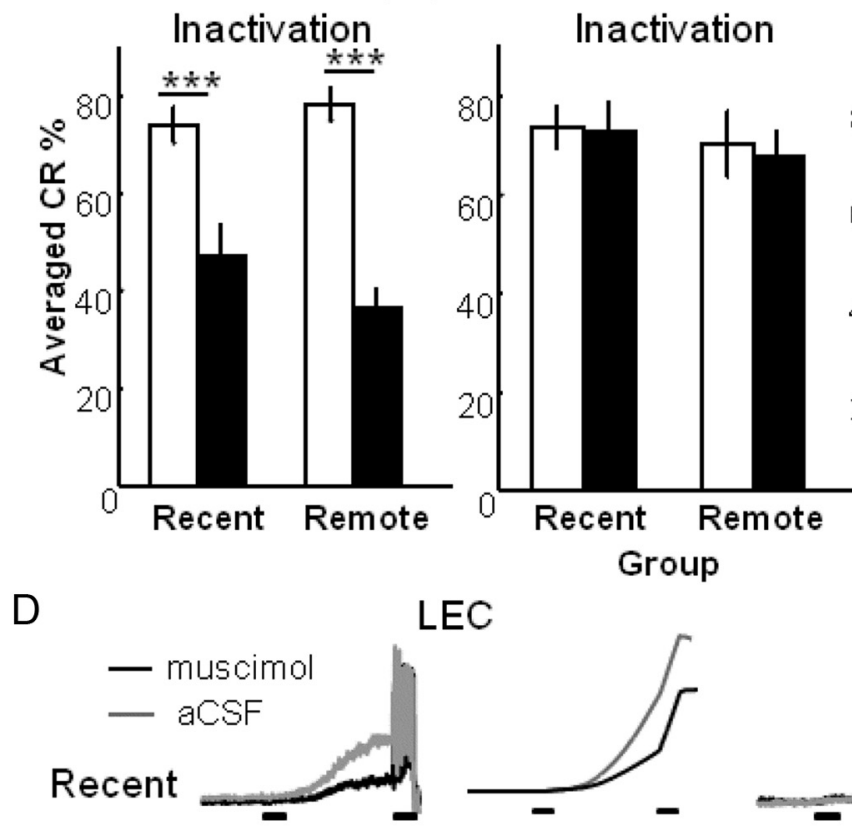

LEC
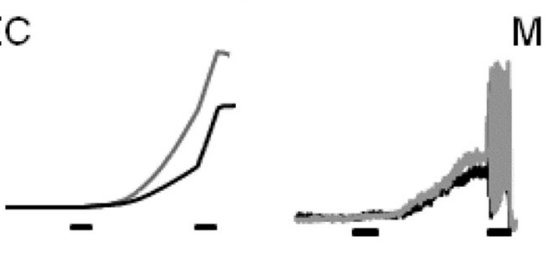

MEC
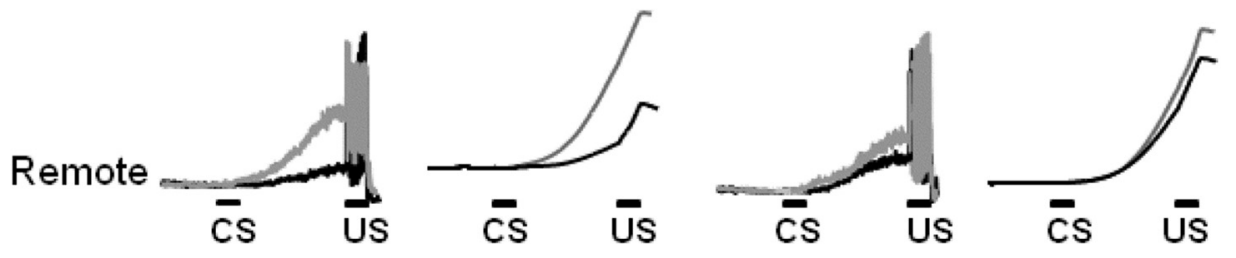

Figure 2. Design, acquisition, and retention in trace conditioning. $\boldsymbol{A}$, Rats were trained in the trace paradigm and tested $1 \mathrm{~d}$ (Recent group) or 1 month (Remote group) after training. $\boldsymbol{B}$, Conditioning sessions consisted of a conditioned stimulus (CS, $100 \mathrm{~ms}$ tone) paired with an unconditioned stimulus (US, $100 \mathrm{~ms}$ periorbital shock) separated by a $500 \mathrm{~ms}$ stimulus-free interval. Animals' eyeblink responses were monitored by recording electromyogram (EMG) in obicularis occuli (top). Approximately 15 of 100 trials were discarded because of hyperactivity before the CS presentation (bottom). Before conditioning, two adaptation sessions (Adt.) recorded spontaneous eyeblinks in the absence of CS or US. The Recent and Remote groups acquired the conditioned response (CR), but were not different from one another. C, Muscimol infusion into the lateral entorhinal cortex (LEC, black bar) severely impaired CR expression compared with aCSF infusion (white bar) at recent $(n=8)$ and remote $(n=10)$ time-points (left). No effect was observed after muscimol infusion into the MEC (center) at either time-point (Recent, $n=6$; Remote, $n=6)$. Combined muscimol infusion into the LEC and MEC impaired CR expression compared with aCSF infusion at recent $(n=6)$ and remote $(n=6)$ test times (right). ${ }^{* *} p<0.01$, ${ }^{* * *} p<0.001 . D$, The increase in averaged amplitude of EMG and the integrated area of the CR following CS onset during sessions with muscimol infusion (black line) into the LEC (left) was smaller than that during sessions with aCSF infusion into either region (gray line) or muscimol infusion into the MEC (right) at both recent (top) and remote (bottom) time points.

Eyeblink conditioning

Conditioning apparatus. Rats were placed in clear cylindrical Plexiglas containers and into one of four sound- and light-attenuating conditioning chambers (Med Associates). Lightweight cables were attached to the pins of the connector secured to the animals head for recording EMG activity and shock delivery. A speaker on the chamber ceiling presented the CS, a $100 \mathrm{~ms}$ tone $(2.5 \mathrm{kHz}, 85 \mathrm{~dB})$. The US was a $100 \mathrm{~ms}$ periorbital shock ( $100 \mathrm{~Hz}$ square pulses). In trace eyeblink conditioning, a stimulusfree trace interval ( $500 \mathrm{~ms}$ ) was interposed between CS offset and US onset. In delay eyeblink conditioning, the CS was extended to $350 \mathrm{~ms}$ 
and coterminated with the US. The US intensity was initiated at 0.3 $\mathrm{mA}$ and carefully calibrated throughout sessions to ensure the minimal current was provided to elicit an eyeblink/head-turn response, which was monitored via web-cameras mounted inside the chambers. The presentations of the CS and US were controlled by a microcomputer (BasicX, Netmedia).

Behavioral procedure. Conditioning began no less than $3 \mathrm{~d}$ after electrode surgery ( $7 \mathrm{~d}$ after the implantation of electrodes and cannulae). The acquisition sessions took place once per day for $12 \mathrm{~d}$ during the dark phase of the reversed light/dark cycle between 1:00 and 5:00 P.M. Days 1-2 allowed rats to acclimate to the procedures in the absence of the CS or US while the frequency of spontaneous eyeblinks was recorded. Presentations of the CS and US began on day 3. Each session consisted of a 10 min acclimation period followed by 100 trials of CS-US pairings over a 50 min span, during which EMG activity was continuously recorded from the obicularis occuli muscle with a RZ-5 recording system (TuckerDavis Technologies). EMG activity was filtered between 0.3 and $3 \mathrm{kHz}$ and digitized at $6 \mathrm{kHz}$. Trials were grouped into 10 trial blocks, with nine paired CS-US presentations followed by one CS only trial. Intervals between trials were pseudorandomized between 20 and $40 \mathrm{~s}$ with a mean of $30 \mathrm{~s}$. Retention sessions followed exactly the same protocol as acquisition sessions.

\section{EMG analysis}

Similar to a previous study (Takehara et al., 2003), amplitude of EMG activity (EMG amplitude) was calculated by subtracting the minimum from maximum EMG signals in a $1 \mathrm{~ms}$ window. A threshold was determined by calculating the mean + SD of EMG amplitude during the 300 ms window before CS onset over 100 trials (including both trials with a CS-US pairing and trials with CS alone presentation). For each trial, EMG amplitudes above this defined threshold were averaged during the $300 \mathrm{~ms}$ period before CS onset ( prevalue) and the $200 \mathrm{~ms}$ period before US onset [conditioned response (CR) value]. Trials were discarded for hyperactivity when the prevalue exceeded $30 \%$ of the threshold $(\sim 15$ of 100 trials). If the $\mathrm{CR}$ value exceeded $5 \times$ the prevalue, the trial was defined as containing a CR. A CR percentage was calculated for each animal for each session as the ratio of CR trials to the total number of valid trials. For visual confirmation, we calculated the averaged EMG amplitude and integrated area of the CR for the $900 \mathrm{~ms}$ period beginning $300 \mathrm{~ms}$ before CS onset until US onset across rats. The integrative area of the CR constitutes the cumulative summation of the averaged EMG signals minus the prevalue for all valid trials.

\section{Histology}

Upon completion of all behavioral tests, rats received intraperitoneal injections of an excess amount of sodium pentobarbital $(150 \mathrm{mg} / \mathrm{kg}$ body weight, Euthansol, Merck) and perfused intracardially with $0.9 \%$ saline, followed by $10 \%$ phosphate-buffered formalin. After cryoprotection, brain tissue was sectioned horizontally at $50 \mu \mathrm{m}$ thickness, mounted on slides, and stained with cresyl violet. Cannula positioning was then identified using a light microscope.

\section{Muscimol spread analysis}

To confirm the spread of muscimol around the injected site, a fluorescent tagged muscimol (muscimol BODIPY TMR-X conjugate, Invitrogen) was infused bilaterally into the LEC and MEC of a few selected rats. Forty minutes after infusion, the rats were killed and perfused. Tissue was sectioned in a coronal plane at $50 \mu \mathrm{m}$ thickness, mounted on slides and imaged with a fluorescent microscope (Zeiss) with a DsRed filter cube. Images were captured with a Retiga EXi camera (Q-Imaging), which was coupled to the microscope, and imaging software (Volocity, PerkinElmer).

\section{Statistical analyses}

Data are expressed as the mean \pm SEM. To determine statistical significance, we used two-way repeated-measures ANOVA with sessions or treatments as a within-subjects factor and retention intervals as a between-subjects factor. When appropriate, paired-samples $t$ tests were used. Criterion for significance was set at $p<0.05$.

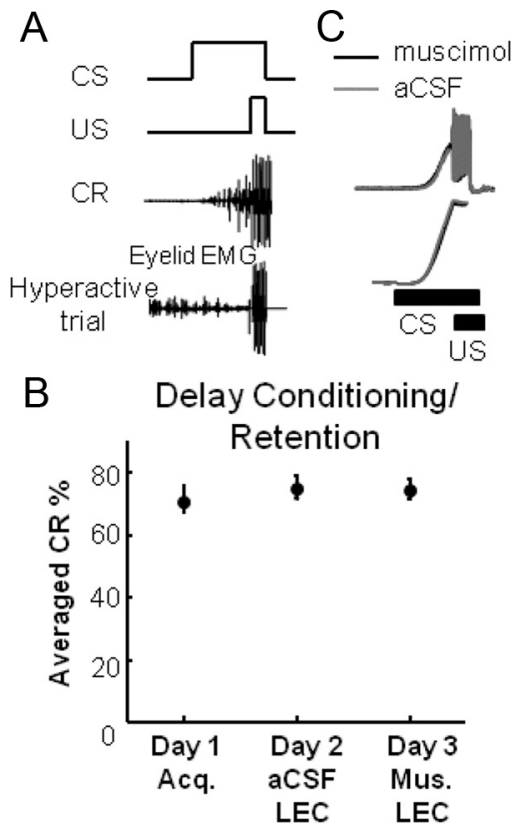

Figure 3. Design, acquisition, and retention in delay conditioning. $\boldsymbol{A}$, Following retention sessions in trace conditioning, the rats received one acquisition session (Acq.) and two retention sessions in a delay paradigm where the conditioned stimulus (CS, $350 \mathrm{~ms}$ tone) coterminated with the unconditioned stimulus (US, $100 \mathrm{~ms}$ periorbital shock). $\boldsymbol{B}$, In the session with muscimol (Mus.) infusions into the lateral entorhinal cortex (LEC), rats showed a comparable percentage of the conditioned response $(C R)$ to the session with aCSF infusion $(n=14)$. C, The temporal pattern of averaged EMG amplitude or integrated area of the CR following CS onset did not differ between sessions with Muscimol infusion (black line) and sessions with aCSF infusion (gray line).

\section{Results}

\section{Histology and muscimol spread analysis}

Histological results confirmed that most cannulae were implanted in their target positions (Fig. 1). A total of 18 rats had cannula in the LEC in both hemispheres. Of these 18 rats, a total of 12 rats also had cannulae bilaterally within the MEC. All animals in which both cannulae were not correctly positioned in the LEC were removed from further analysis.

Upon completion of behavioral trainings, four rats were injected with a conjugate of muscimol and the BODIPY TMR-X fluorophore. The fluorescent signals were detected specifically in the regions of interest, and spread was confined to a maximal $1 \mathrm{~mm}$ range (Fig. 1A), consistent with a previous study (Allen et al., 2008).

\section{Effects of LEC and MEC inactivation on the memory retrieval of an association between temporally discontiguous stimuli} Either $1 \mathrm{~d}$ (Recent group) or 1 month (Remote group) after the 10 th acquisition session in trace eyeblink conditioning, memory retention was tested with infusion of either muscimol or aCSF into the LEC, MEC, or both (Fig. 2A). All rats acquired the conditioned response over the course of acquisition, with no differences between groups (Fig. $2 B$; two-way repeated-measures ANOVA; session, $F_{(11,176)}=83.876, p<0.0001$; group, $F_{(1,16)}=$ 2.139 , n.s.; interaction, $F_{(11,176)}=0.525$, n.s. $)$.

During retention sessions $1 \mathrm{~d}$ after the last acquisition session (Recent group), infusion of muscimol into the LEC significantly impaired the expression of CRs compared with aCSF infusion. A similar impairment was observed in the Remote group, when muscimol was infused into the LEC before testing retention of the memory acquired 1 month earlier (Fig. $2 C$; two-way repeatedmeasures ANOVA; infusion, $F_{(1,16)}=80.512, p<0.001$; group, 
$F_{(1,16)}=0.414$, n.s.; interaction, $F_{(1,16)}=3.624$, n.s. $)$. Conversely, there were no effects of muscimol infusion into the MEC on the expression of CRs either $1 \mathrm{~d}$ or 1 month after training (infusion, $F_{(1,10)}=0.85$, n.s.; group, $F_{(1,10)}=2.336$, n.s.; interaction, $F_{(1,16)}=$ 2.921. n.s.). In addition, inactivation of both the LEC and MEC produced no further impairment on the expression of CRs than inactivation of the LEC alone (infusion into the LEC only or both the LEC and MEC, $F_{(1,10)}=0.198$, n.s.; group, $F_{(1,10)}=0.718$, n.s.; interaction, $F_{(1,10)}=1.809$, n.s.).

Visual inspection of EMG activity confirmed a reduction of CR expression following muscimol infusion into the LEC (Fig. $2 D)$. In all sessions with aCSF infusion or muscimol infusion into the MEC, the averaged EMG amplitude and integrated area of the CR gradually increased from CS offset to US onset. This increase was much smaller in sessions with muscimol infusion into the LEC. Finally, infusion had no effect on trial selection, as the average number of trials removed each session (because of hyperactivity) did not differ between the LEC muscimol infusion (mean $=15.1 \pm 1.35$ ) and the LEC aCSF infusion sessions $\left(\right.$ mean $=15.22 \pm 1.44 ; t_{(17)}=-.113$, n.s. $)$.

\section{Effects of LEC inactivation on the memory retrieval of an association between temporally contiguous stimuli}

To confirm that the observed effects of LEC inactivation were specific to the association between discontiguous stimuli, following trace eyeblink retention sessions, all animals were trained and tested in delay eyeblink conditioning, where the CS coterminated with the US (Fig. 3A). Delay conditioning consisted of $1 \mathrm{~d}$ of training, followed by $2 \mathrm{~d}$ of retention where aCSF or muscimol were infused into the LEC. In the session with muscimol infusion, the rats exhibited a comparable percentage of CRs to the session with aCSF infusion (Fig. $3 B$, paired-samples $t$ test, $t_{(13)}=0.376$, n.s.). The averaged EMG amplitude and integrated area of the $\mathrm{CR}$ across trials also did not differ between the session with aCSF infusion and the session with muscimol infusion (Fig. $3 C$ ). These results suggest that the effect of LEC inactivation was specific to memory expression in trace eyeblink conditioning, in which an association must be formed between discontiguous stimuli.

\section{Discussion}

We revealed that inactivation of the LEC, but not the MEC, impaired memory expression in trace eyeblink conditioning, in which rats were required to form an association between temporally discontiguous stimuli. The observed impairment could not be attributed to impairments in sensory processing or motor coordination, because LEC inactivation had no effect on memory expression in delay eyeblink conditioning involving the same stimuli and behavioral monitors, but no imposed interval between the stimuli.

Consistent with previous research (Otto and Eichenbaum, 1992; Ryou et al., 2001; Esclassan et al., 2009), the present results reveal an important contribution of the entorhinal cortex to mnemonic processes during paradigms requiring the maintenance of sensory information over a temporal delay. Theoretical models (Hasselmo et al., 2000; Hasselmo and Stern, 2006) have related this function of the entorhinal cortex to its self-sustaining spike activity observed in vitro (Egorov et al., 2002; Tahvildari et al., 2007) and persistent activity during a delay period following stimulus presentation in vivo (Suzuki et al., 1997; Young et al., 1997). Although persistent activity can be observed in both the LEC and MEC (Egorov et al., 2002; Tahvildari et al., 2007), the LEC is more strongly connected with the perirhinal cortex, whose projections mediate highly processed sensory information (Fur- tak et al., 2007), and with the prelimbic medial prefrontal cortex (Hoover and Vertes, 2007; Agster and Burwell, 2009), which plays a critical role in memory retrieval in trace eyeblink conditioning (Takehara-Nishiuchi et al., 2005; Oswald et al., 2010). Furthermore, these two regions show differential selectivity for spatial versus nonspatial information (Hargreaves et al., 2005). These differences in anatomical connectivity between the LEC and MEC may explain the observed functional dissociation in the trace paradigm, whereby the LEC may function to maintain CS information during the trace interval by sustained neuronal activity, enabling hippocampal or prefrontal networks to recover patterns of neuronal activities selective for the previously acquired association (McEchron and Disterhoft, 1997; Takehara-Nishiuchi and McNaughton, 2008). Targeted manipulations to self-sustaining entorhinal activity using a muscarinic or metabotropic glutamate receptor antagonist (Klink and Alonso, 1997; Yoshida et al., 2008; Esclassan et al., 2009) could test this possibility.

The hippocampus plays a critical role in forming an association between two stimuli with a temporal delay (Moyer et al., 1990; McEchron et al., 1998; Chowdhury et al., 2005), however, its involvement in memory expression is timelimited (Kim et al., 1995; Takehara et al., 2003; Quinn et al., 2008). In contrast, we found a long-lasting role for the LEC in memory retrieval, as LEC inactivation impaired expression of both recent (1-d-old) and remote (1-month-old) memories in trace eyeblink conditioning. This is consistent with findings of long-lasting retrograde memory impairments when large hippocampal lesions include the rhinal cortices in humans (Bayley et al., 2006) and primates (Suzuki et al., 1993), and with specific lesions to subregions of the rhinal cortices in rodents (Cho and Kesner, 1996; Burwell et al., 2004). Together, these findings suggest that the rhinal cortices play a long-lasting role in memory storage or retrieval. In a spatial memory task, however, entorhinal involvement in memory expression has been shown to decrease 6 weeks after training (Cho and Kesner, 1996), suggesting that the rhinal cortices may be involved in a more extended consolidation process than the hippocampus and may eventually disengage from the network supporting memory storage and expression. Future electrophysiological studies will help discriminate these two possibilities by examining activity profiles of entorhinal neurons in the context of interaction with other neocortical regions that are presumed to store permanent memory.

In conclusion, the present study demonstrates a long-lasting role for the LEC in memory retrieval for an association between temporally discontiguous stimuli, providing further support for a functional dissociation between the LEC and MEC.

\section{References}

Agster KL, Burwell RD (2009) Cortical efferents of the perirhinal, postrhinal, and entorhinal cortices of the rat. Hippocampus 19:1159-1186.

Allen TA, Narayanan NS, Kholodar-Smith DB, Zhao Y, Laubach M, Brown TH (2008) Imaging the spread of reversible brain inactivations using fluorescent muscimol. J Neurosci Methods 171:30-38.

Bayley PJ, Hopkins RO, Squire LR (2006) The fate of old memories after medial temporal lobe damage. J Neurosci 26:13311-13317.

Burwell RD, Bucci DJ, Sanborn MR, Jutras MJ (2004) Perirhinal and postrhinal contributions to remote memory for context. J Neurosci 24:11023-11028.

Canto CB, Wouterlood FG, Witter MP (2008) What does the anatomical organization of the entorhinal cortex tell us? Neural Plast 381243:1-18.

Cho YH, Kesner RP (1996) Involvement of the entorhinal cortex or parietal cortex in long-term spatial discrimination memory in rats: Retrograde amnesia. Behav Neurosci 110:436-442.

Chowdhury N, Quinn JJ, Fanselow MS (2005) Dorsal hippocampus in- 
volvement in trace fear conditioning with long, but not short, trace intervals in mice. Behav Neurosci 119:1396-1402.

Egorov AV, Hamam BN, Fransén E, Hasselmo ME, Alonso AA (2002) Graded persistent activity in entorhinal cortex neurons. Nature 420: 173-178.

Esclassan F, Coutureau E, Di Scala G, Marchand AR (2009) A cholinergicdependent role for the entorhinal cortex in trace fear conditioning. J Neurosci 29:8087-8093.

Furtak SC, Wei SM, Agster KL, Burwell RD (2007) Functional neuroanatomy of the parahippocampal region in the rat: the perirhinal and postrhinal cortices. Hippocampus 17:709-722.

Fyhn M, Molden S, Witter MP, Moser EI, Moser MB (2004) Spatial representation in the entorhinal cortex. Science 305:1258-1264.

Gaskin S, White NM (2010) Temporary inactivation of the dorsal entorhinal cortex impairs acquisition and retrieval of spatial information. Neurobiol Learn Mem 93:203-207.

Hafting T, Fyhn M, Molden S, Moser MB, Moser EI (2005) Microstructure of a spatial map in the entorhinal cortex. Nature 436:801-806.

Hargreaves EL, Rao G, Lee I, Knierim JJ (2005) Major dissociation between medial and lateral entorhinal input to dorsal hippocampus. Science 308:1792-1794.

Hasselmo ME, Stern CE (2006) Mechanisms underlying working memory for novel information. Trends Cogn Sci 10:487-493.

Hasselmo ME, Fransen E, Dickson C, Alonso AA (2000) Computational modeling of entorhinal cortex. Ann N Y Acad Sci 911:418-446.

Hoover WB, Vertes RP (2007) Anatomical analysis of afferent projections to the medial prefrontal cortex in the rat. Brain Struct Funct 212:149-179.

Kim JJ, Clark RE, Thompson RF (1995) Hippocampectomy impairs the memory of recently, but not remotely, acquired trace eyeblink conditioned responses. Behav Neurosci 109:195-203.

Klink R, Alonso A (1997) Muscarinic modulation of the oscillatory and repetitive firing properties of entorhinal cortex layer II neurons. J Neurophysiol 77:1813-1828.

McEchron MD, Disterhoft JF (1997) Sequence of single neuron changes in CA1 hippocampus of rabbits during acquisition of trace eyeblink conditioned responses. J Neurophysiol 78:1030-1044.

McEchron MD, Bouwmeester H, Tseng W, Weiss C, Disterhoft JF (1998) Hippocampectomy disrupts auditory trace fear conditioning and contextual fear conditioning in the rat. Hippocampus 8:638-646.

Moyer JR Jr, Deyo RA, Disterhoft JF (1990) Hippocampectomy disrupts trace eye-blink conditioning in rabbits. Behav Neurosci 104:243-252.

Oswald BB, Maddox SA, Tisdale N, Powell DA (2010) Encoding and retrieval are differentially processed by the anterior cingulate and prelimbic cortices: a study based on trace eyeblink conditioning in the rabbit. Neurobiol Learn Mem 93:37-45.
Otto T, Eichenbaum H (1992) Complementary roles of the orbital prefrontal cortex and the perirhinal-entorhinal cortices in an odor-guided delayed-nonmatching-to-sample task. Behav Neurosci 106:762-775.

Paxinos G, Watson C (1998) The rat brain in stereotaxic coordinates, Ed 4. San Diego: Academic.

Quinn JJ, Ma QD, Tinsley MR, Koch C, Fanselow MS (2008) Inverse temporal contributions of the dorsal hippocampus and medial prefrontal cortex to the expression of long-term fear memories. Learn Mem 15:368-372.

Ryou JW, Cho SY, Kim HT (2001) Lesions of the entorhinal cortex impair acquisition of hippocampal-dependent trace conditioning. Neurobiol Learn Mem 75:121-127.

Scoville WB, Milner B (1957) Loss of recent memory after bilateral hippocampal lesions. J Neurol Neurosurg Psychiatry 20:11-21.

Squire LR, Zola-Morgan S (1991) The medial temporal lobe memory system. Science 253:1380-1386.

Steffenach HA, Witter M, Moser MB, Moser EI (2005) Spatial memory in the rat requires the dorsolateral band of the entorhinal cortex. Neuron 45:301-313.

Suzuki WA, Zola-Morgan S, Squire LR, Amaral DG (1993) Lesions of the perirhinal and parahippocampal cortices in the monkey produce longlasting memory impairment in the visual and tactual modalities. J Neurosci 13:2430-2451.

Suzuki WA, Miller EK, Desimone R (1997) Object and place memory in the macaque entorhinal cortex. J Neurophysiol 78:1062-1081.

Tahvildari B, Fransén E, Alonso AA, Hasselmo ME (2007) Switching between "On" and "Off" states of persistent activity in lateral entorhinal layer III neurons. Hippocampus 17:257-263.

Takehara K, Kawahara S, Kirino Y (2003) Time-dependent reorganization of the brain components underlying memory retention in trace eyeblink conditioning. J Neurosci 23:9897-9905.

Takehara-Nishiuchi K, McNaughton BL (2008) Spontaneous changes of neocortical code for associative memory during consolidation. Science 322:960-963.

Takehara-Nishiuchi K, Kawahara S, Kirino Y (2005) NMDA receptordependent processes in the medial prefrontal cortex are important for acquisition and the early stage of consolidation during trace, but not delay eyeblink conditioning. Learn Mem 12:606-614.

Yoshida M, Fransén E, Hasselmo ME (2008) mGluR-dependent persistent firing in entorhinal cortex layer III neurons. Eur J Neurosci 28:11161126.

Young BJ, Otto T, Fox GD, Eichenbaum H (1997) Memory representation within the parahippocampal region. J Neurosci 17:5183-5195. 